\title{
BIODIVERSITY OF CYANOBACTERIA IN FRESH WATER PONDS OF PUDUKKOTTAI DISTRICT, TAMILNADU, INDIA
}

\author{
J. Dhanalakshmi 1, P. Jeevan 1, E. Angel Jemima ${ }^{2}$,
}

${ }^{1}$ Department of Microbiology, J. J. College of Arts \& Science (Autonomous), J. J. Nagar, Sivapuram, Pudukkottai - 622422.

2 Trichy Research Institute of Biotechnology, No.107-D, $2^{\text {nd }}$ floor, $9^{\text {th }}$ cross (East), Thillai nagar, Trichy - 620018, Tamil Nadu, India.

\begin{abstract}
Cyanobacterial species (blue-green algae) constitute the major part of the phytoplanktonic biomass during the summer in freshwater ponds. The aim of the research work was to study the biodiversity of cyanobacteria among 20 different freshwater ponds of the Pudukkottai district of Tamil Nadu, India. The morphological identification of cyanobacterial species was carried out using a trinocular microscope. The results showed that the maximum number of cyanobacterial species belonged to Oscillatoriaceae, Nostocaceae, Microcystaceae, Scenedesmaceae, and Desmidiaceae families. Among 25 different families of Cyanobacteria about 42 distinct species were identified. These results showed that the freshwater ponds of the Pudukkottai district have an abundance of cyanobacteria species.
\end{abstract}

Keywords: Biodiversity, Cyanobacteria, Oscillatoriaceae, Nostocaceae, Microcystaceae, fresh water ponds. 


\section{Introduction}

Fresh water ponds enclose a specific ecosystem with respect to the pond setting, and it's comprised of numerous plants, aquatic animals and bacteria [1]. Each element of freshwater pond's ecosystem is always dependent on the other elements and organisms for their survival The primary producers of fresh water ponds are phytoplanktons, the secondary are zooplankton and the ecosystem also contains decomposers [2, 3]. Cyanobacteria (blue-green algae) are widely distributed in fresh water ponds and are capable of performing both carbon assimilation and $\mathrm{N}_{2}$ fixation, thereby enhancing productivity in a variety of environments. They secrete a number of biologically active substances rather than nitrogen fixation [4]. Tropical conditions prevailing in India provide a favorable environment for the abundant growth of these organisms in the fresh water ecosystems $[5,6]$. They constitute the greater part of the phytoplanktonic biomass during the summer [7,8]. Certain species of Cyanobacteria in the genera Anabaena, Aphanizomenon, Microcystis, and Oscillatoria most often form extensive and persistent blooms in freshwater aquaculture ponds [9]. The development of Cyanobacterial blooms is favored under conditions when the availability of nitrogen is limited compared to phosphorus, and in warm water temperatures. Under those conditions, dominance of phytoplankton communities by cyanobacteria is the result of certain unique physiological attributes (in particular, $\mathrm{N}_{2}$ fixation and buoyancy regulation) that allow Cyanobacteria to compete effectively with other phytoplankton. The ability to regulate cell buoyancy through environmentally-controlled collapse and reformation of intracellular gas vacuoles is perhaps the primary reason for the frequent dominance of aquaculture pond phytoplankton communities by Cyanobacteria [10],[11].

Cyanobacteria are always found in close association with a diverse array of microorganisms, including eubacteria, fungi, and protozoans [12]. Cyanobacteria are very important organisms for the health and growth of many plants. Cyanobacteria play an important role in the nitrogen cycle. They are one of very few groups of organisms that can convert inert atmospheric nitrogen into an organic form, such as nitrate or ammonia and also they provide oxygen as a by-product of photosynthesis [13], a food source for other organisms such as zooplankton, insects and snails, and in the case of larger filamentous algae, a habitat for small animals [14]. The most common toxic Cyanobacteria in fresh water are Microcystis spp., Cylindrospermopsis raciborskii, Planktothrix (syn. Oscillatoria) rubescens, Synechococcus spp., Planktothrix (syn. Oscillatoria) agardhii, Gloeotrichia spp., Anabaena spp., Lyngbya spp., Aphanizomenon spp., Nostoc spp., some Oscillatoria spp., Schizothrix spp. and Synechocystis spp. This study presents the isolation and characterization of different types of Cyanobacterial species in fresh water ponds of Pudukkottai district, Tamil Nadu, India.

\section{Materials and methods}

\subsection{Collection of samples}

There are 20 different ponds in a different monthly intervals for a period ranging from Aug 2018 to Sep 2019 in Pudukkottai dictrict located in Tamil Nadu, India. Pond water samples were collected in sterilized bottles of $500 \mathrm{ml}$ capacity at a depth of about $30 \mathrm{~cm}$. The sample is fixed soon after collected and taken to the laboratory for analysis. 


\subsection{Morphological identification of Cyanobacteria}

Morphological identification of Cyanobacteria was done by spreading a Cyanobacterial culture on glass slides using forceps. Culture were covered with glass cover slips and observed their size, shape, color and other features under low (10X) and high power (100 X) objective lens of Trinocular microscope.

\subsection{Species identification of Cyanobacteria}

Various species of Cyanobacteria present in fresh water ponds of Pudukkottai district were identified using the reference manual Cyanophyta by T.V. Desikachary and "Manual of freshwater algae of Tamil Nadu’by G. Mahendra Perumal and N. Anand (2009).

\section{Results and discussion}

\subsection{Isolation \& identification of Cyanobacteria in fresh water ponds}

Cyanobacterial species present in fresh water ponds of Pudukkottai district were identified and classified based on the reference manual. Nearly 25 different families of Cyanobacteria species were identified has been diverse in the fresh water ponds of Pudukkottai District. About 42 versatile species of Cyanobacteria belonging to 25 different families were found to reside in the freshwater ecosystem of Pudukkottai district. Each species has its own unique size and shape which distinguishes them to be classified under various families. Each and every species has its own significance in the ecosystem in which they live.

\subsection{Species identification of Cyanobacteria}

Anabaena spbaerica, Anabaenopsis arnoldii (aptekarj) and Microcystis aeruginosa were identified in fresh water ponds of the Thirumalaisamuthiram Kathirkameshvarar temple [PW 1] and Gloeocapsa nigrescens was isolated in Santhanatha swami temple [PW 2], Pudukkottai. Chroococcus turgidus, Microcystis robusta was present in Peraiyur -naganathar swami temple [PW 3]. Species named Arthrospira platensis was found to reside in the fresh water pond located in Kulathur- Varatharaja Perumal temple [PW 4]. Various species of Cyanobacteria like Oscillatoria princeps, Anabena iyengarii, Nostoc linckia, Cylindrospermu stagnale, Cosmarium quadrum lund, cosmorium subalatum, Cosmorium sexnotatum, Staurastrum pantanale sp.nov were found in the fresh water of the Pudukkottai Sri Brahadhambal temple [PW 5]. About 10 different species of Cyanobacteria like Lyngbya majuscule, Lyngbya wollei, Ulothrix sp., Fragilaria crotomensis (Kitton), synedra dorsiventralis (ehrenberg), Westella linearis GM smith, Scenedesmus acuminatus, Scendesmus denticulatus (lagerheim), Scendesmus vijugatus, Cosmorium subprotumidum resides in ThiruvarankulamArangulanathar temple [PW 6]. Haematococcus lacustris, Symploca jurassica was identified in PW 7 \& PW 8 respectively. Fresh water pond [PW 9] in Thirumayam- Kottai sathyamoorthy perumal temple contains Merismopedia glauca, Aphanocapsa pulchra (kutz) and Merismopedia punctata Meyen. 
Species named Spirulina subsalsa, Cylindrospermum stagnale, Closterium lunula (Mull) was isolated in PW 10, PW 11 and PW 12 respectively and no Cyanobacterial species were identified in the fresh water pond of Vadavaalam- Kaliyuga meyya ayyanar temple [PW 13]. Ceratium hirundinella was identified in the PW 14. In the fresh water pond of MalaiyurPeriyanayaki amman temple [PW 15], species like Chlorococcum bumicola, Navicula capitatoradiata, Mougeotia scalaris and Chlorella was identified. PW 16 has distribution of Cyanobacterial species Lyngbya aestuarii, Lyngbya majuscula, Oscillatoria nigra and Oscillatoria curviceps.In PW 17 only Cylindrocapsa geminella was identified. Five different species of nitzschia obtusa, Microcystis aeruginosa, Microcystis flos-aquae, Microcystis robusta and Navicula capitatoradiata were present in PW 18. Fresh water ponds in Ramar temple [PW 19] and Koothandaramar temple [PW 20] has distribution of Nostoc calcicola and Spirogyra subsalsa respectively as shown in Table 1 .

Table 1. Identification of Cyanobacterial species from 20 different fresh water ponds of Pudukkottai District

\begin{tabular}{|c|c|c|c|c|}
\hline S. No & $\begin{array}{l}\text { Pond } \\
\text { water }\end{array}$ & $\begin{array}{l}\text { Name of the pond } \\
\text { place }\end{array}$ & $\begin{array}{l}\text { Longitude \& } \\
\text { Lattitude }\end{array}$ & Name of algae \\
\hline 1. & PW 1 & $\begin{array}{l}\text { Thirumalai } \\
\text { chamuthiram- } \\
\text { Kathirkameshvarar } \\
\text { temple. }\end{array}$ & $\begin{array}{ll}10^{0} 21^{\prime} 19^{\prime}, & \mathrm{N}, \\
78^{0} 49^{\prime} 42^{\prime}, \mathrm{E} & \end{array}$ & $\begin{array}{l}\text { Anabaena spbaerica } \\
\text { Anabaenopsis arnoldii } \\
\text { (aptekarj), } \\
\text { Microcystis aeruginosa }\end{array}$ \\
\hline 2. & PW 2 & $\begin{array}{l}\text { Pudukkottai } \\
\text { Santhanatha swami } \\
\text { temple }\end{array}$ & $\begin{array}{l}10.3802^{0} \\
78.8135^{0} \mathrm{E}\end{array}$ & Gloeocapsa nigrescens \\
\hline 3. & PW 3 & $\begin{array}{l}\text { Peraiyur -naganathar } \\
\text { swami temple. }\end{array}$ & $\begin{array}{l}10.3528^{0} \\
78.7564^{0} \mathrm{E}\end{array}$ & $\begin{array}{l}\text { Chroococcus turgidus } \\
\text { Microcystis robusta }\end{array}$ \\
\hline 4. & PW 4 & $\begin{array}{l}\text { Kulathur- Varatharaja } \\
\text { Perumal temple. }\end{array}$ & $\begin{array}{l}10.7002892^{0} \quad \mathrm{~N}, \\
78.5470346^{0} \mathrm{E}\end{array}$ & Arthrospira platensis \\
\hline 5. & PW 5 & $\begin{array}{l}\text { Pudukkottai-Sri } \\
\text { Brahadhambal } \\
\text { temple. }\end{array}$ & $\begin{array}{l}10.3915^{0} \\
78.8005^{0} \mathrm{E}\end{array}$ & $\begin{array}{l}\text { Oscillatoria princeps } \\
\text { Anabena iyengarii } \\
\text { Nostoc linckia } \\
\text { Cylindrospermum stagnale } \\
\text { Cosmarium quadrum lund }\end{array}$ \\
\hline
\end{tabular}




\begin{tabular}{|c|c|c|c|c|}
\hline & & & & $\begin{array}{l}\text { Cosmorium subalatum } \\
\text { Cosmorium sexnotatum } \\
\text { Staurastrum pantanale } \\
\text { sp.nov }\end{array}$ \\
\hline 6. & PW 6 & $\begin{array}{l}\text { Thiruvarankulam- } \\
\text { Arangulanathar } \\
\text { temple }\end{array}$ & $\begin{array}{l}10.3561^{0} \\
78.8737^{0} \mathrm{E}\end{array}$ & $\begin{array}{l}\text { Lyngbya majuscule } \\
\text { Lyngbya wollei } \\
\text { Ulothrix sp. } \\
\text { Fragilaria crotomensis } \\
\text { (Kitton) } \\
\text { Synedra dorsiventralis } \\
\text { (ehrenberg) } \\
\text { Westella linearis GM smith } \\
\text { Scenedesmus acuminatus } \\
\text { Scendesmus denticulatus } \\
\text { (lagerheim) } \\
\text { Scendesmus vijugatus } \\
\text { Cosmorium subprotumidum }\end{array}$ \\
\hline 7. & PW 7 & $\begin{array}{l}\text { Kumaramalai - } \\
\text { Balathandayuthabani }\end{array}$ & $\begin{array}{l}10^{0} 21^{\prime} 53^{\prime}, \\
78^{0} 43^{\prime} 39^{\prime}, \mathrm{N}\end{array}$ & Haematococcus lacustris \\
\hline 8. & PW 8 & $\begin{array}{l}\text { Thirumayam- } \\
\text { sathyagireeswarar } \\
\text { temple }\end{array}$ & $\begin{array}{l}10.2471^{0} \\
78.750481^{0} \mathrm{E}\end{array}$ & Symploca jurassica \\
\hline 9. & PW 9 & $\begin{array}{l}\text { Thirumayam- Kottai } \\
\text { sathyamoorthy } \\
\text { perumal temple. }\end{array}$ & $\begin{array}{l}10.2471^{0} \\
78.7508^{0} \mathrm{E}\end{array}$ & $\begin{array}{l}\text { Merismopedia glauca } \\
\text { Aphanocapsa pulchra (kutz) } \\
\text { Merismopedia punctata } \\
\text { Meyen }\end{array}$ \\
\hline 10 . & PW 10 & $\begin{array}{l}\text { Pudukkotai } \\
\text { Chellayiamman } \\
\text { temple. }\end{array}$ & $\begin{array}{l}10^{0} 22^{\prime} 33^{\prime}, \\
78^{0} 49^{\prime} 12^{\prime}, \mathrm{E}\end{array}$ & Spirulina subsalsa \\
\hline
\end{tabular}




\begin{tabular}{|c|c|c|c|c|}
\hline 11. & PW 11 & $\begin{array}{l}\text { Keeranur- Lord } \\
\text { Iyyappan temple. }\end{array}$ & $\begin{array}{l}10.571564^{0} \quad \mathrm{~N}, \\
78.784416^{0} \mathrm{E}\end{array}$ & Cylindrospermum stagnale \\
\hline 12. & PW 12 & $\begin{array}{l}\text { Alankudi- } \\
\text { Chithivinayagar } \\
\text { temple. }\end{array}$ & $10.37^{0} \mathrm{~N}, 78.89^{0} \mathrm{E}$ & Closterium lunula (Mull) \\
\hline 13. & PW 13 & $\begin{array}{l}\text { Vadavaalam- } \\
\text { Kaliyuga meyya } \\
\text { ayyanar temple. }\end{array}$ & $\begin{array}{ll}10^{0} 25^{\prime} 37^{\prime}, & \mathrm{N}, \\
78^{0} 53^{\prime} 58^{\prime}, \mathrm{E} & \end{array}$ & Nil \\
\hline 14. & PW 14 & $\begin{array}{l}\text { Thirumanancheri- } \\
\text { Periyanayaki ambika } \\
\text { samayathaya suganthi } \\
\text { parimaleshwarar } \\
\text { temple. }\end{array}$ & $\begin{array}{l}11.099957^{0} \quad \mathrm{~N}, \\
79.556036 \mathrm{E}\end{array}$ & Ceratium hirundinella \\
\hline 1 & PW 15 & $\begin{array}{l}\text { Malaiyur- } \\
\text { Periyanayaki amman } \\
\text { temple. }\end{array}$ & $\begin{array}{l}10.359119 \quad \mathrm{~N}, \\
78.985173 \mathrm{E}\end{array}$ & $\begin{array}{l}\text { Chlorococcum bumicola } \\
\text { Navicula capitatoradiata } \\
\text { Mougeotia scalaris } \\
\text { Chlorella. }\end{array}$ \\
\hline 16. & PW 16 & $\begin{array}{l}\text { Varappur- } \\
\text { Agathishwarar } \\
\text { temple. }\end{array}$ & $\begin{array}{l}10^{0} 21^{\prime} 2^{\prime}, \\
78^{0} 28^{\prime} 42^{\prime}, \mathrm{N}\end{array}$ & $\begin{array}{l}\text { Lyngbya aestuarii } \\
\text { Lyngbya majuscula } \\
\text { Oscillatoria nigra } \\
\text { Oscillatoria curviceps }\end{array}$ \\
\hline 17. & PW 17 & $\begin{array}{l}\text { Ichadi- Vinayagar } \\
\text { temple }\end{array}$ & $\begin{array}{l}10.4254^{0} \quad \mathrm{~N}, \\
78.8800^{0} \mathrm{E}\end{array}$ & Cylindrocapsa geminella \\
\hline 18. & PW 18 & $\begin{array}{l}\text { Sempattividuthi- } \\
\text { Vinayagar temple. }\end{array}$ & $\begin{array}{l}1004288^{0} \\
78.9776^{0} \mathrm{E}\end{array}$ & $\begin{array}{l}\text { Nitzschia obtusa } \\
\text { Microcystis aeruginosa } \\
\text { Microcystis flos-aquae } \\
\text { Microcystis robusta } \\
\text { Navicula capitatoradiata }\end{array}$ \\
\hline 19. & PW 19 & $\begin{array}{l}\text { Ramachandrapuram- } \\
\text { Ramar temple. }\end{array}$ & $\begin{array}{l}10.244036^{0} \quad \mathrm{~N}, \\
78.747511^{0} \mathrm{E}\end{array}$ & Nostoc calcicola \\
\hline
\end{tabular}




\begin{tabular}{|l|l|l|l|l|}
\hline 20. & PW 20 & $\begin{array}{l}\text { Thuvar- } \\
\text { Koothandaramar } \\
\text { temple. }\end{array}$ & $\begin{array}{l}10^{0} 34^{\prime} 17^{\prime}, \quad \text { N, } \\
79^{0} 0^{\prime} 57^{\prime}, \mathrm{E}\end{array}$ & Spirogyra subsalsa \\
\hline
\end{tabular}

\subsection{Family identification of Cyanobacteria}

The identified Cyanobacterial species belongs to 25 different families. The majority of species was found in the family Oscillatoriaceae, Nostocaceae, Microcystaceae, Scenedesmaceae and Desmidiaceae. The family Desmidiaceae includes microalgal species like Cosmarium quadrum lund, Cosmorium subprotumidum, cosmorium subalatum, Cosmorium sexnotatum, Staurastrum pantanale sp.nov. The Cyanobacterial species like Oscillatoria princeps, Lyngbya majuscule, Lyngbya wollei, lyngbya aestuarii, lyngbya majuscula, Oscillatoria nigra, Oscillatoria curviceps were found to classified under the family Oscillatoriaceae. Also the family Nostocaceae including species like Nostoc linckia, Nostoc calcicola, Cylindrospermum stagnale, Anabaena spbaerica and Anabena iyengarii was isolated in the fresh water ponds of Pudukkottai district. The pond ecosystem also found to contain various species of family Microcystaceae which includes Microcystis aeruginosa, Microcystis marginata, Microcystis marginata, Microcystis aeruginosa, Microcystis aeruginosa, Microcystis flos-aqua, Microcystis flos-aquae and Microcystis robusta. The distribution of species like Scenedesmus acuminatus, Scendesmus denticulatus (lagerheim), Scendesmus vijugatus, Scenedesmus quadricauda of family Scenedesmaceae were found in the freshwater ponds were shown in Table 2.

Table 1. Identification of Cyanobacterial species from 20 different fresh water ponds of Pudukkottai District

\begin{tabular}{|l|l|l|l|l|}
\hline S. No & $\begin{array}{l}\text { Pond } \\
\text { water }\end{array}$ & $\begin{array}{l}\text { Name of the pond } \\
\text { place }\end{array}$ & $\begin{array}{l}\text { Longitude \& } \\
\text { Lattitude }\end{array}$ & Name of algae \\
\hline 1. & PW 1 & $\begin{array}{l}\text { Thirumalai } \\
\text { chamuthiram- } \\
\text { Kathirkameshvarar } \\
\text { temple. }\end{array}$ & $\begin{array}{l}10^{0} 21^{\prime} 19^{\prime}, \quad \mathrm{N}, \\
78^{0} 49^{\prime} 42^{\prime \prime} \mathrm{E}\end{array}$ & $\begin{array}{l}\text { Anabaena spbaerica } \\
\text { Anabaenopsis arnoldii } \\
\text { (aptekarj), } \\
\text { Microcystis aeruginosa }\end{array}$ \\
\hline 2. & PW 2 & $\begin{array}{l}\text { Pudukkottai } \\
\text { Santhanatha swami } \\
\text { temple }\end{array}$ & $\begin{array}{l}10.3802^{0} \\
78.8135^{\circ} \mathrm{E}\end{array}$ & $\mathrm{N}$, \\
\hline 3. & PW 3 & $\begin{array}{l}\text { Peraiyur -naganathar } \\
10.3528^{0}\end{array}$ & $\mathrm{~N}$, & Chroococcus turgidus \\
\hline
\end{tabular}




\begin{tabular}{|c|c|c|c|c|}
\hline & & swami temple. & $78.7564^{0} \mathrm{E}$ & Microcystis robusta \\
\hline 4. & PW 4 & $\begin{array}{l}\text { Kulathur- Varatharaja } \\
\text { Perumal temple. }\end{array}$ & $\begin{array}{l}10.7002892^{0} \quad \mathrm{~N}, \\
78.5470346^{0} \mathrm{E}\end{array}$ & Arthrospira platensis \\
\hline 5. & PW 5 & $\begin{array}{l}\text { Pudukkottai-Sri } \\
\text { Brahadhambal } \\
\text { temple. }\end{array}$ & $\begin{array}{l}10.3915^{0} \quad \mathrm{~N}, \\
78.8005^{0} \mathrm{E}\end{array}$ & $\begin{array}{l}\text { Oscillatoria princeps } \\
\text { Anabena iyengarii } \\
\text { Nostoc linckia } \\
\text { Cylindrospermum stagnale } \\
\text { Cosmarium quadrum lund } \\
\text { Cosmorium subalatum } \\
\text { Cosmorium sexnotatum } \\
\text { Staurastrum pantanale } \\
\text { sp.nov }\end{array}$ \\
\hline 6. & PW 6 & $\begin{array}{l}\text { Thiruvarankulam- } \\
\text { Arangulanathar } \\
\text { temple }\end{array}$ & $\begin{array}{l}10.3561^{0} \\
78.8737^{0} \mathrm{E}\end{array}$ & $\begin{array}{l}\text { Lyngbya majuscule } \\
\text { Lyngbya wollei } \\
\text { Ulothrix sp. } \\
\text { Fragilaria crotomensis } \\
\text { (Kitton) } \\
\text { Synedra dorsiventralis } \\
\text { (ehrenberg) } \\
\text { Westella linearis GM smith } \\
\text { Scenedesmus acuminatus } \\
\text { Scendesmus denticulatus } \\
\text { (lagerheim) } \\
\text { Scendesmus vijugatus } \\
\text { Cosmorium subprotumidum }\end{array}$ \\
\hline 7. & PW 7 & $\begin{array}{l}\text { Kumaramalai - } \\
\text { Balathandayuthabani }\end{array}$ & $\begin{array}{l}10^{0} 21^{\prime} 53^{\prime}, \quad \mathrm{N}, \\
78^{0} 43^{\prime} 39^{\prime}, \mathrm{E}\end{array}$ & Haematococcus lacustris \\
\hline
\end{tabular}




\begin{tabular}{|c|c|c|c|c|}
\hline 8. & PW 8 & $\begin{array}{l}\text { Thirumayam- } \\
\text { sathyagireeswarar } \\
\text { temple }\end{array}$ & $\begin{array}{ll}10.2471^{0} & \mathrm{~N}, \\
78.750481^{0} \mathrm{E} & \end{array}$ & Symploca jurassica \\
\hline 9. & PW 9 & $\begin{array}{l}\text { Thirumayam- Kottai } \\
\text { sathyamoorthy } \\
\text { perumal temple. }\end{array}$ & $\begin{array}{l}10.2471^{0} \quad \mathrm{~N}, \\
78.7508^{0} \mathrm{E}\end{array}$ & $\begin{array}{l}\text { Merismopedia glauca } \\
\text { Aphanocapsa pulchra (kutz) } \\
\text { Merismopedia punctata } \\
\text { Meyen }\end{array}$ \\
\hline 10 . & PW 10 & $\begin{array}{l}\text { Pudukkotai } \\
\text { Chellayiamman } \\
\text { temple. }\end{array}$ & $\begin{array}{l}10^{0} 22^{\prime} 33^{\prime}, \\
78^{0} 49^{\prime} 12^{\prime}, \mathrm{E}\end{array}$ & Spirulina subsalsa \\
\hline 11. & PW 11 & $\begin{array}{l}\text { Keeranur- Lord } \\
\text { Iyyappan temple. }\end{array}$ & $\begin{array}{l}10.571564^{0} \quad \mathrm{~N}, \\
78.784416^{0} \mathrm{E}\end{array}$ & Cylindrospermum stagnale \\
\hline 12. & PW 12 & $\begin{array}{l}\text { Alankudi- } \\
\text { Chithivinayagar } \\
\text { temple. }\end{array}$ & $10.37^{0} \mathrm{~N}, 78.89^{0} \mathrm{E}$ & Closterium lunula (Mull) \\
\hline 13. & PW 13 & $\begin{array}{l}\text { Vadavaalam- } \\
\text { Kaliyuga meyya } \\
\text { ayyanar temple. }\end{array}$ & $\begin{array}{l}10^{0} 25^{\prime} 37^{\prime}, \\
78^{0} 53^{\prime} 58^{\prime \prime} \mathrm{E}\end{array}$ & Nil \\
\hline 14. & PW 14 & $\begin{array}{l}\text { Thirumanancheri- } \\
\text { Periyanayaki ambika } \\
\text { samayathaya suganthi } \\
\text { parimaleshwarar } \\
\text { temple. }\end{array}$ & $\begin{array}{l}11.099957^{0} \\
79.556036 \mathrm{E}\end{array}$ & Ceratium hirundinella \\
\hline 1 & PW 15 & $\begin{array}{l}\text { Malaiyur- } \\
\text { Periyanayaki amman } \\
\text { temple. }\end{array}$ & $\begin{array}{l}10.359119 \\
78.985173 \mathrm{E}\end{array}$ & $\begin{array}{l}\text { Chlorococcum bumicola } \\
\text { Navicula capitatoradiata } \\
\text { Mougeotia scalaris } \\
\text { Chlorella. }\end{array}$ \\
\hline 16. & PW 16 & $\begin{array}{l}\text { Varappur- } \\
\text { Agathishwarar } \\
\text { temple. }\end{array}$ & $\begin{array}{l}10^{0} 21^{\prime} 2{ }^{\prime}, \\
78^{0} 28^{\prime} 422^{\prime}, \mathrm{E}\end{array}$ & $\begin{array}{l}\text { Lyngbya aestuarii } \\
\text { Lyngbya majuscula } \\
\text { Oscillatoria nigra }\end{array}$ \\
\hline
\end{tabular}




\begin{tabular}{|c|c|c|c|c|}
\hline & & & & Oscillatoria curviceps \\
\hline 17. & PW 17 & $\begin{array}{l}\text { Ichadi- } \quad \text { Vinayagar } \\
\text { temple }\end{array}$ & $\begin{array}{l}10.4254^{0} \\
78.8800^{0} \mathrm{E}\end{array}$ & Cylindrocapsa geminella \\
\hline 18. & PW 18 & $\begin{array}{l}\text { Sempattividuthi- } \\
\text { Vinayagar temple. }\end{array}$ & $\begin{array}{l}1004288^{0} \\
78.9776^{0} \mathrm{E}\end{array}$ & $\begin{array}{l}\text { Nitzschia obtusa } \\
\text { Microcystis aeruginosa } \\
\text { Microcystis flos-aquae } \\
\text { Microcystis robusta } \\
\text { Navicula capitatoradiata }\end{array}$ \\
\hline 19. & PW 19 & $\begin{array}{l}\text { Ramachandrapuram- } \\
\text { Ramar temple. }\end{array}$ & $\begin{array}{l}10.244036^{0} \quad \mathrm{~N}, \\
78.747511^{0} \mathrm{E}\end{array}$ & Nostoc calcicola \\
\hline 20. & PW 20 & $\begin{array}{l}\text { Thuvar- } \\
\text { Koothandaramar } \\
\text { temple. }\end{array}$ & $\begin{array}{ll}10^{0} 34^{\prime} 17^{\prime}, & \mathrm{N}, \\
79^{0} 00^{\prime} 57^{\prime}, \mathrm{E} & \end{array}$ & Spirogyra subsalsa \\
\hline
\end{tabular}

\section{Conclusions}

In any ecosystem, not a single species grow independently and indefinitely, because each and every species are interlinked and has cyclic transformation of nutrients. The physicochemical changes in the environment in which they reside might affect particular species and can induce the growth and abundance of other species, which leads to the succession of several species in a period of time. A large number of Cyanobacterial species has been isolated and classified into 42 different species belongs to 25 different families present in the 20 different fresh water ponds of Pudukkottai district, Tamil Nadu, India which shows the abundance of Cyanobacteria in fresh water ponds. 


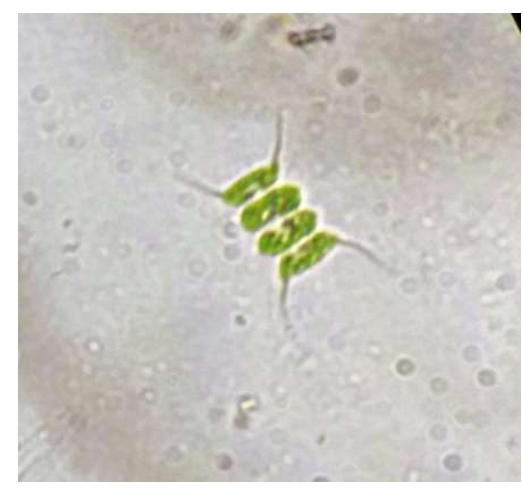

(1)

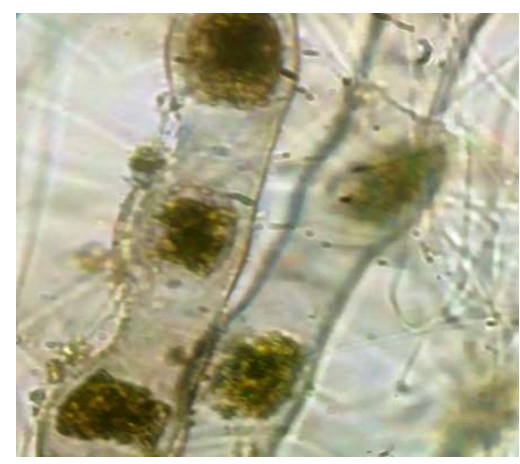

(4)

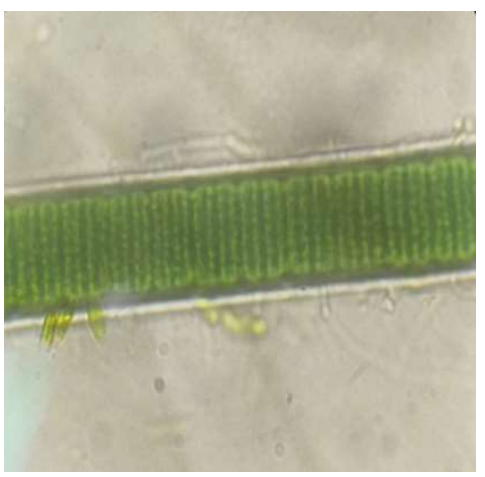

(7)

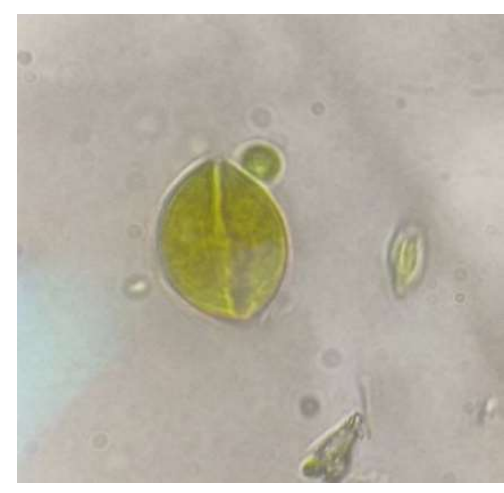

(2)

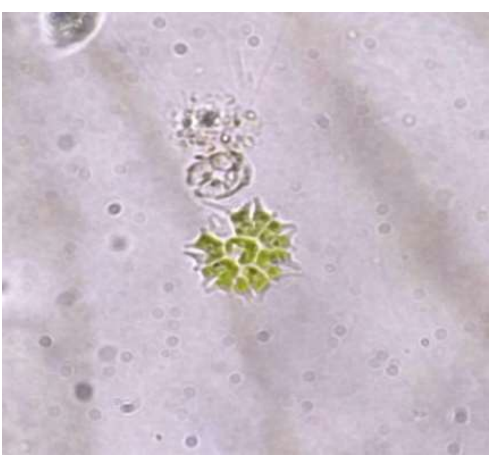

(5)

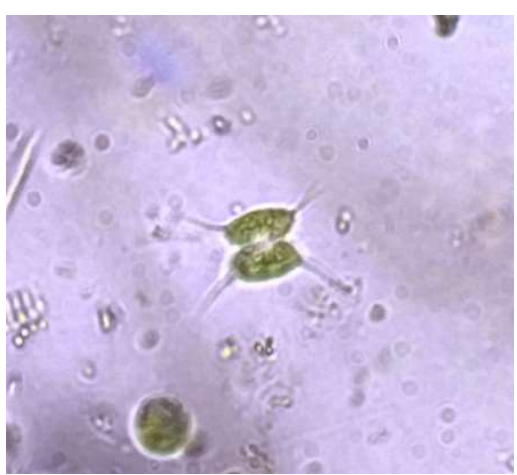

(8)

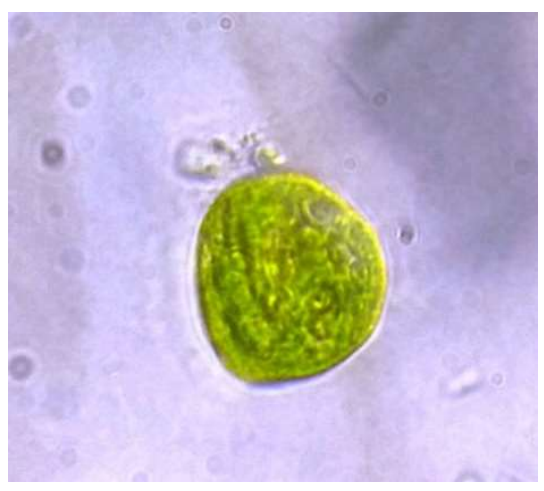

(3)

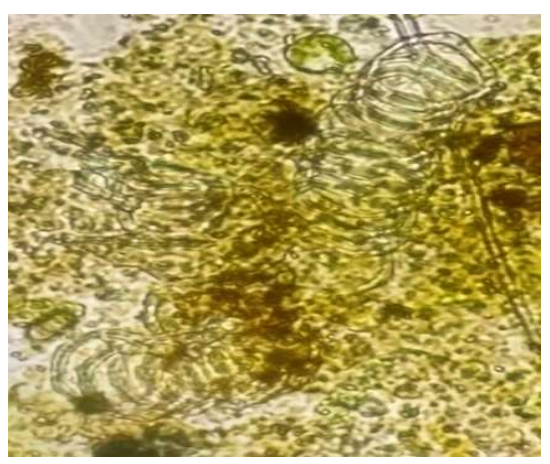

(6)

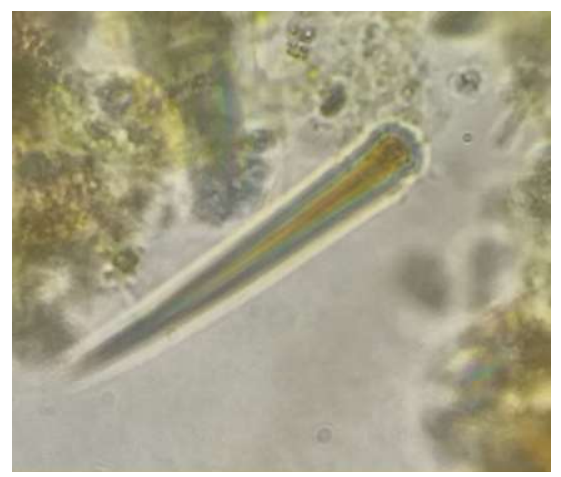

(9) 


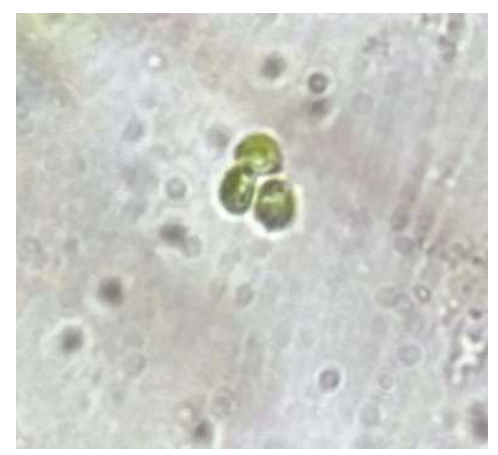

(10)

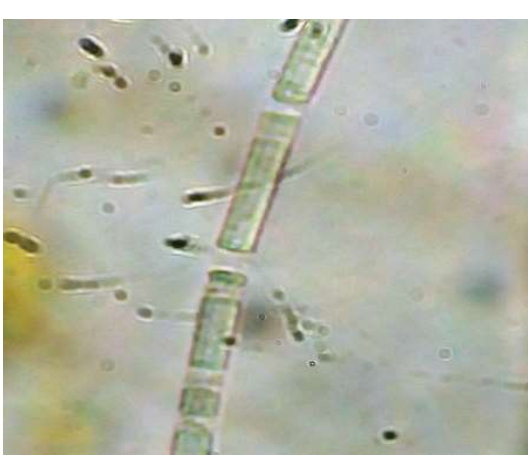

(13)

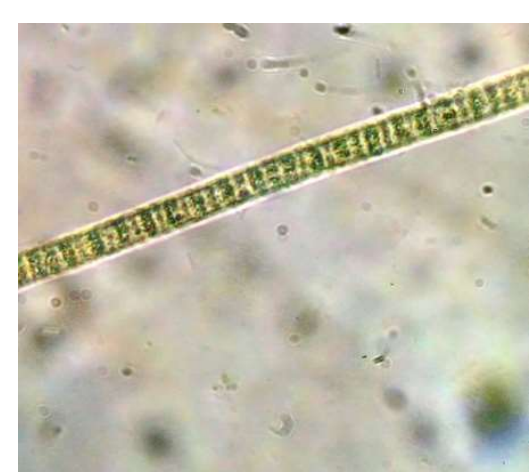

(16)

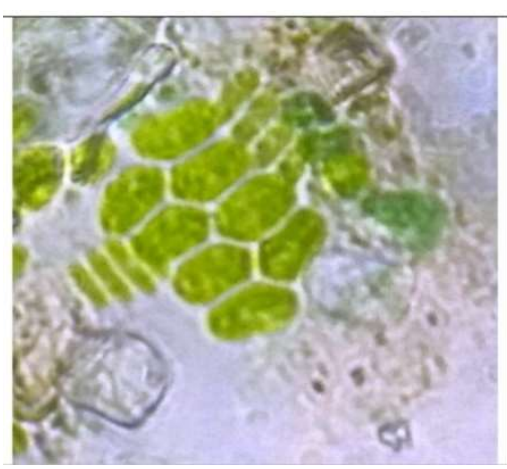

(11)

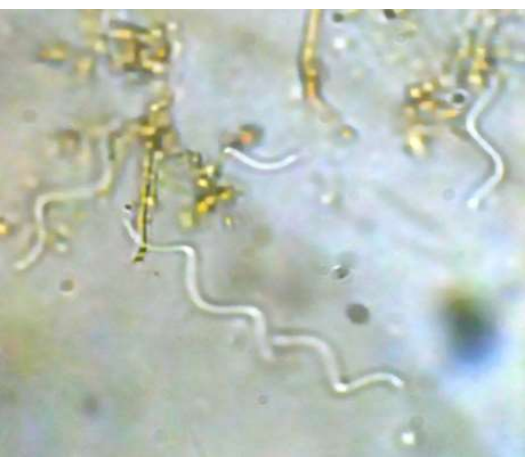

(14)
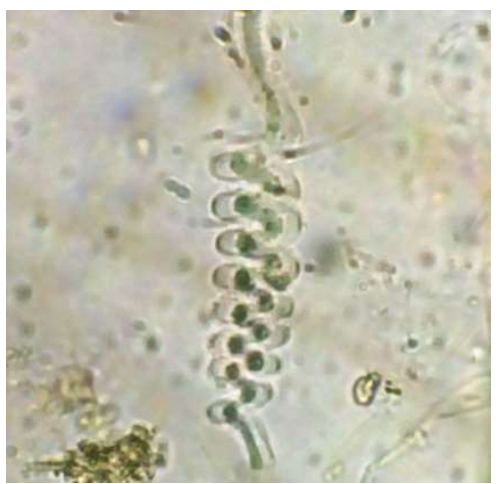

(17)

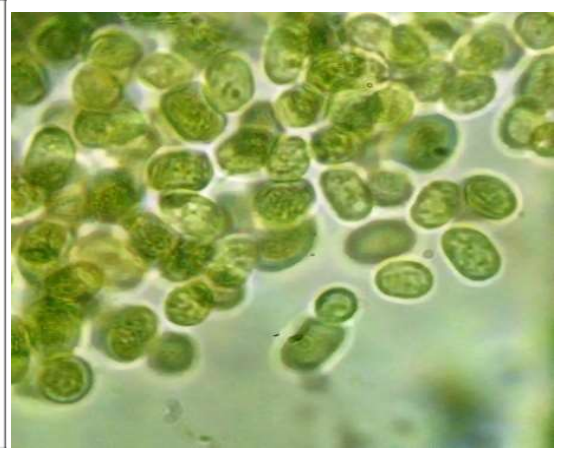

(12)

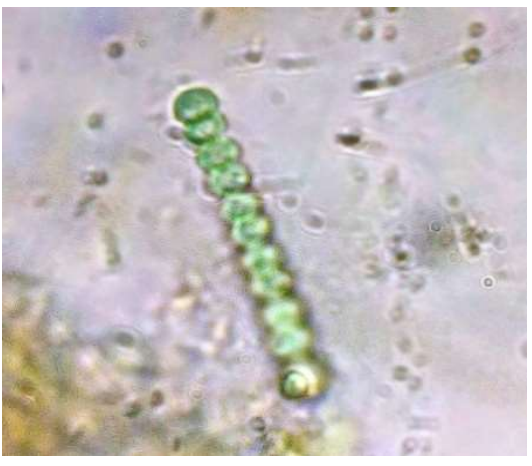

(15)

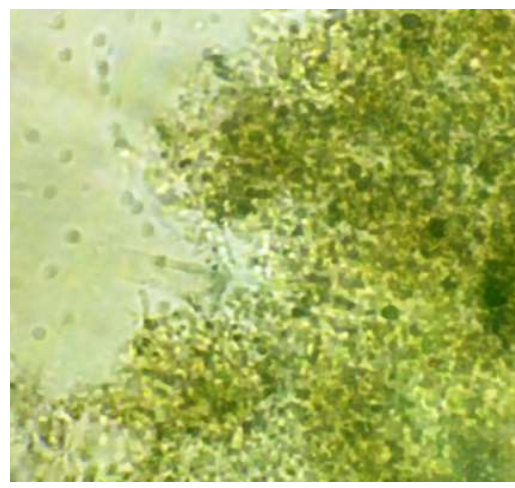

(18) 


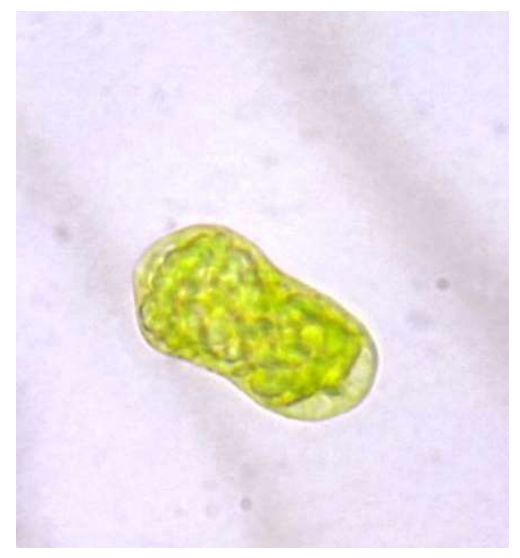

(19)

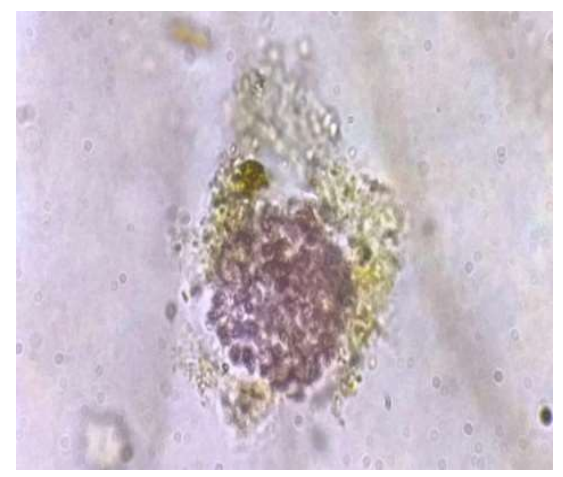

(22)

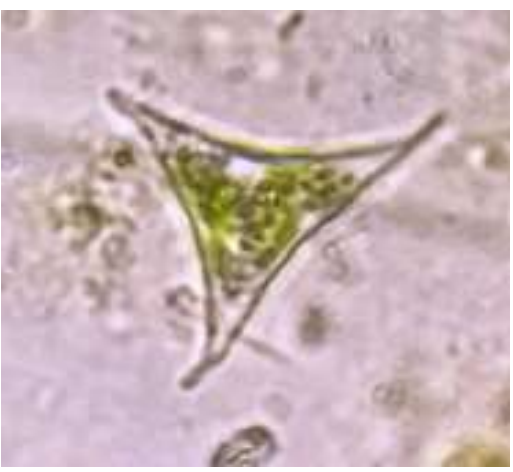

(25)

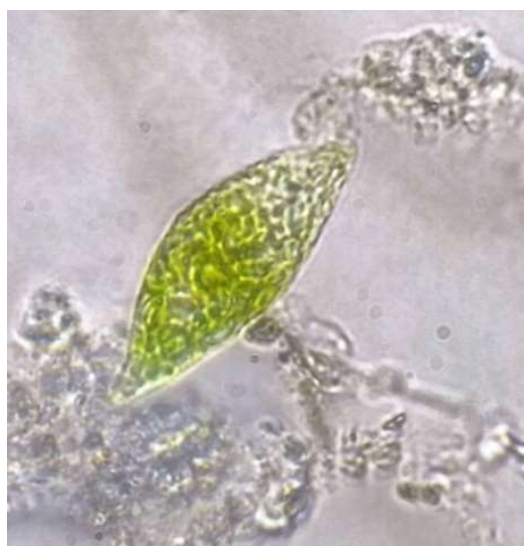

(20)

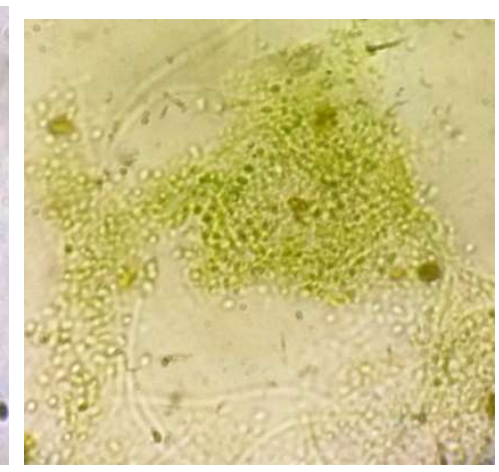

(23)
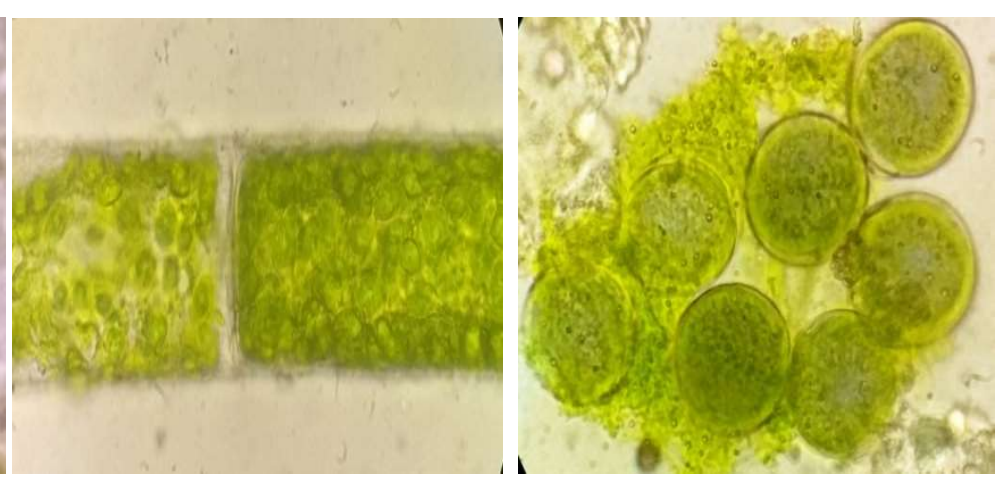

(26)

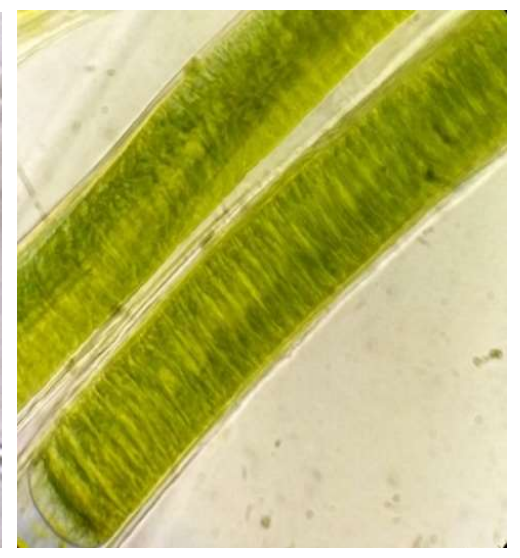

(21)

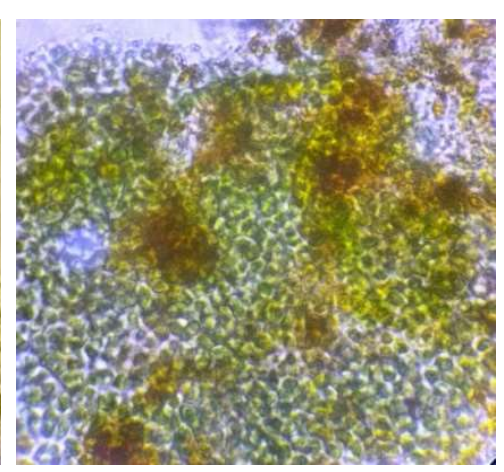

(24) 


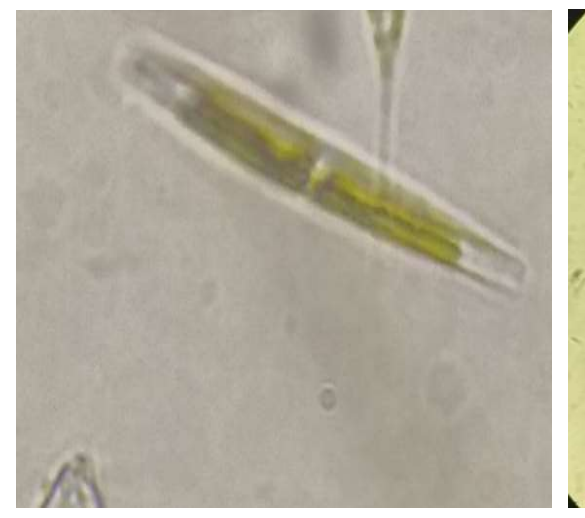

(28)

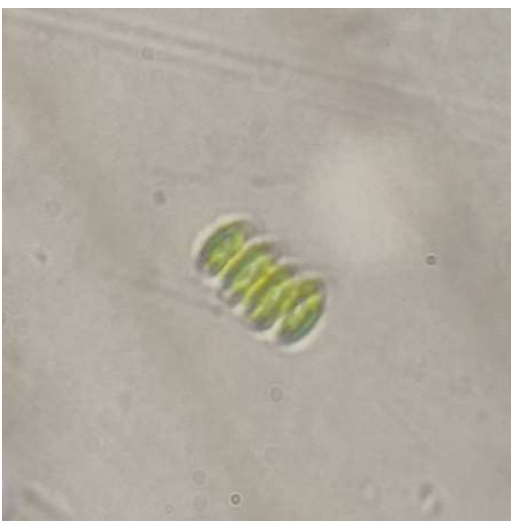

(31)

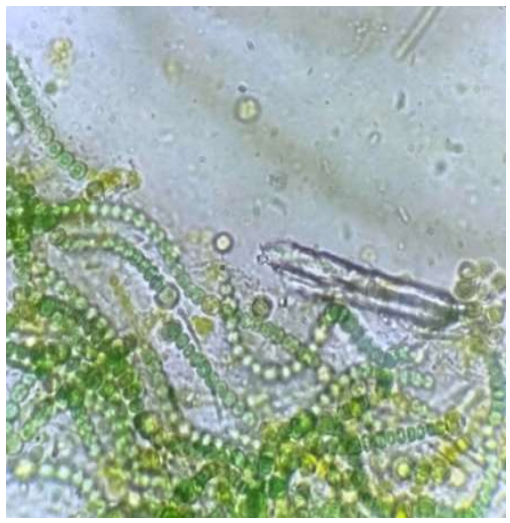

(34)

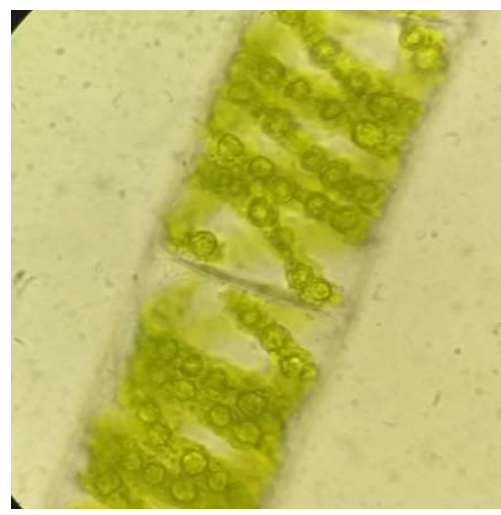

(29)

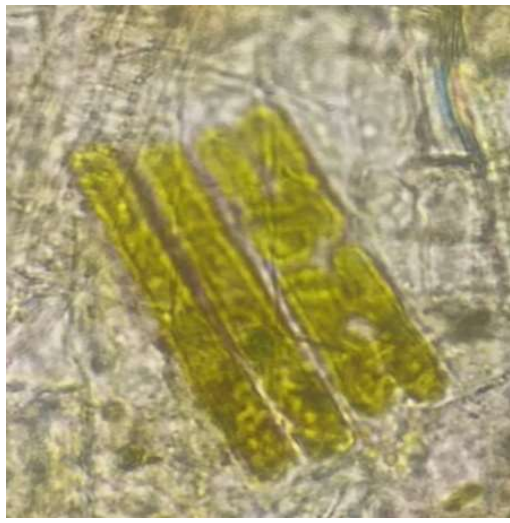

(32)

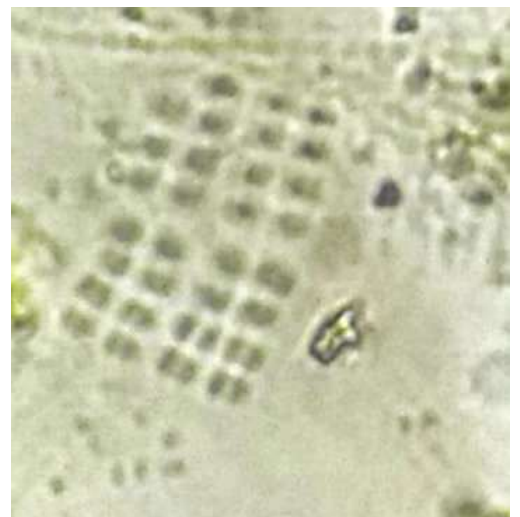

(35)

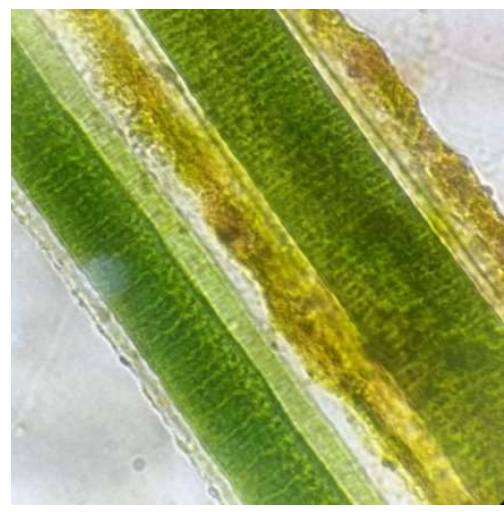

(30)

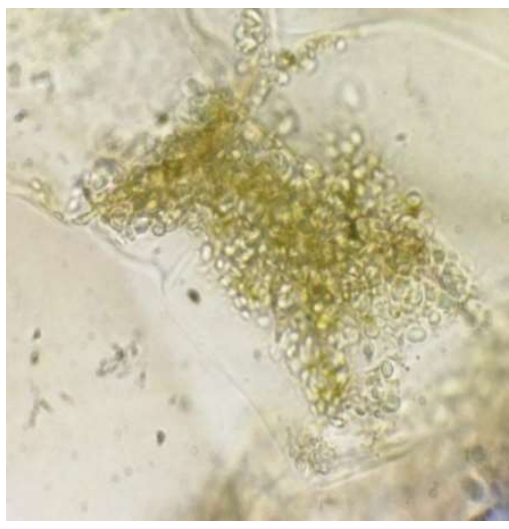

(33)

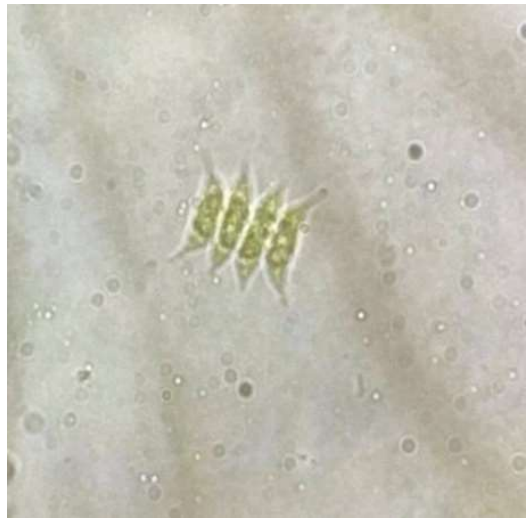

(36) 


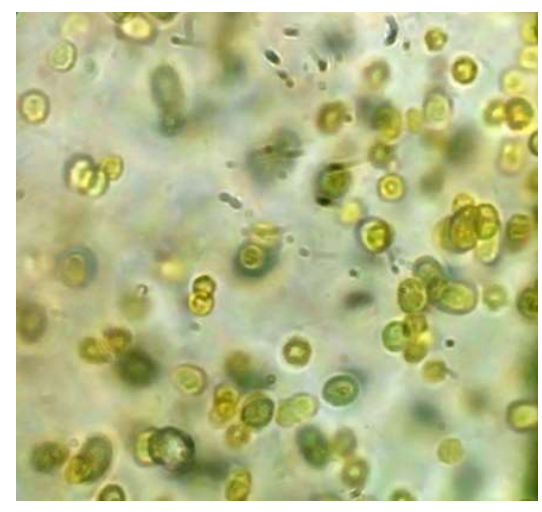

(37)

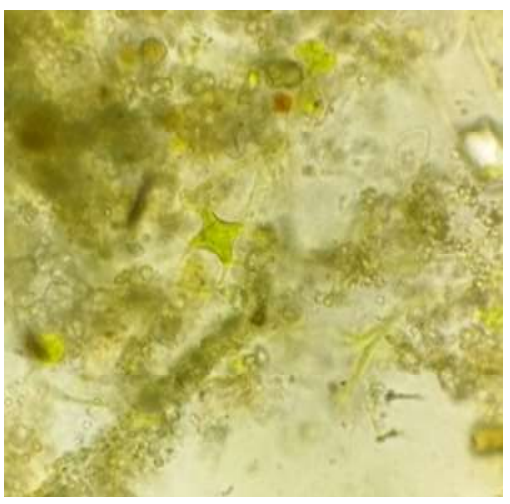

(40)

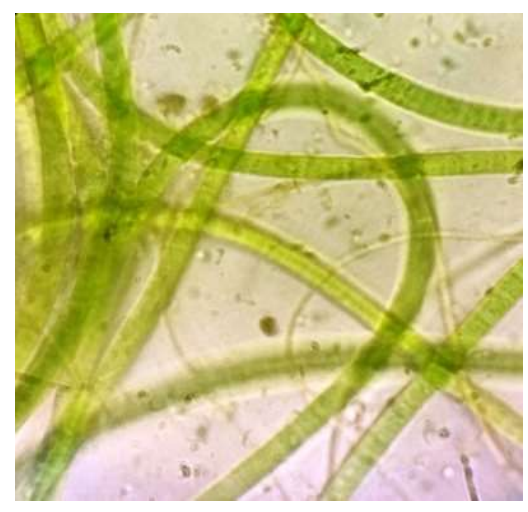

(38)

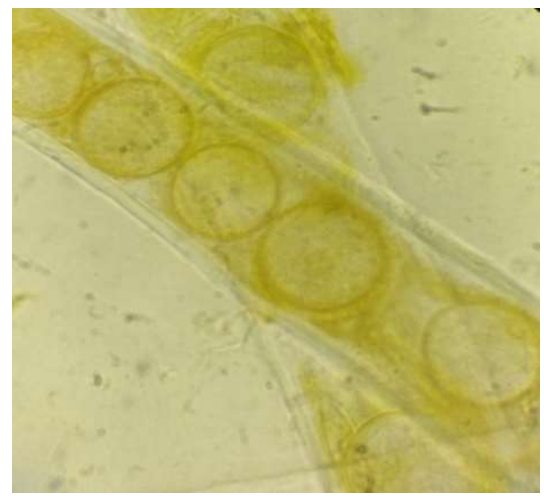

(41)

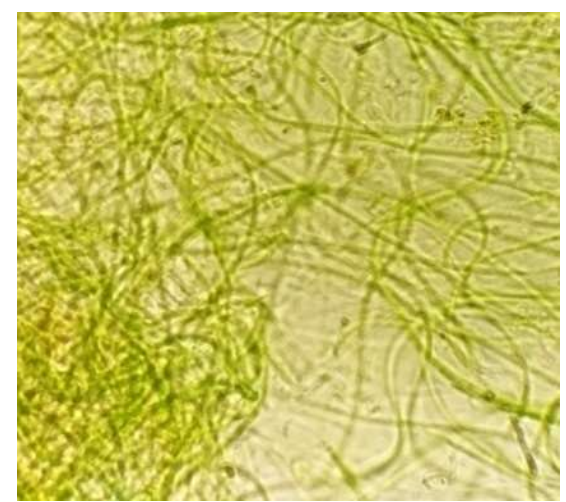

(39)

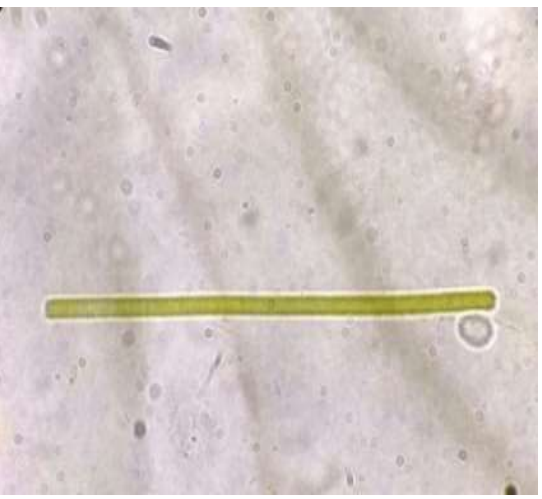

(42)

\section{Figure Legends}

Fig 1. Scenedesmus quadricauda

Fig 2. Cocconeis pediculus

Fig 3. Haematococcus lacustris

Fig 4. Cylindrocapsa geminella

Fig 5. Pediastrum simplex (Meyen) Lemm

Fig 6. Arthrospira platensis

Fig 7. Oscillatoria curviceps

Fig 8. Anabaenopsis arnoldii (aptekarj)

Fig 9. Calothrix spp 
Fig 10. Chroococcus turgidus

Fig 12. Scendesmus vijugatus

Fig 13. Symploca jurassica

Fig 14. Spirulina subsalsa

Fig 15. Nostoc calcicola

Fig 16. Oscillatoria nigra

Fig 17. Arthrospira platensis

Fig 18. Microcystis aeruginosa

Fig 19. Microcystis marginata

Fig 20. Microcystis marginata

Fig 21. Lyngbya majuscula

Fig 22. Microcystis aeruginosa

Fig 23. Microcystis aeruginosa

Fig 24. Microcystis flos-aquae

Fig 25. Staurastrum pantanale sp.nov

Fig 26. Cladophora glomerata (L.)

Fig 27. Chlorococcum bumicola (Naeg ravenhorst)

Fig 28. Closterium lunula (Mull)

Fig 29. Spirogyra subsalsa (Kuetzing)

Fig 30. Lyngbya aestuarii

Fig 31. Scendesmus denticulatus (lagerheim)

Fig 32. Fragilaria crotomensis (Kitton)

Fig 33. Microcystis robusta

Fig 34. Nostoc linckia

Fig 35. Merismopedia punctata Meyen

Fig 36. Scenedesmus acuminatus

Fig 37. Aphanocapsa pulchra (kutz) 
Fig 38. Lyngbya wollei

Fig 39. Ulothrix spp

Fig 40. Ceratium hirundinella

Fig 41. Lyngbya majuscule

Fig 42. Oscillatoria princeps

\section{Table legends}

Table 1. Identification of Cyanobacterial species from 20 different fresh water ponds of Pudukkottai District.

Table 2. Family classification of identified Cyanobacterial species from fresh water ponds

\section{Acknowledgement}

The author thank Trichy Research Institute of Biotechnology for the constant support by providing research facility throughout the study.

\section{Conflict of Interest}

The authors declare no conflict of interest

\section{References}

1. Demoulin, C. F., Lara, Y. J., Cornet, L., François, C., Baurain, D., Wilmotte, A., Javaux, E. J. (2019). Cyanobacteria evolution: Insight from the fossil record. Free radical biology \& medicine, 140, 206-223.

2. Haraldsson, M., Gerphagnon, M., Bazin, P., Colombet, J., Tecchio, S., Sime-Ngando, T., Niquil, N. (2018). Microbial parasites make Cyanobacteria blooms less of a trophic dead end than commonly assumed. The ISME journal, 12(4), 1008-1020.

3. Konstantinou, D., Gerovasileiou, V., Voultsiadou, E., Gkelis, S. (2018). SpongesCyanobacteria associations: Global diversity overview and new data from the Eastern Mediterranean. PloS one, 13(3), e0195001

4. Hilborn, E. D., Beasley, V. R. (2015). One health and Cyanobacteria in freshwater systems: animal illnesses and deaths are sentinel events for human health risks. Toxins, 7(4), 1374-1395.

5. Singh, J. S., Kumar, A., Rai, A. N., Singh, D. P. (2016). Cyanobacteria: a precious bioresource in agriculture, ecosystem, and environmental sustainability. Frontiers in microbiology, 7, 529. 
6. Issa, A. A., Abd-Alla, M. H., Ohyama, T. (2014). Nitrogen fixing Cyanobacteria: future prospect. Advances in biology and ecology of nitrogen fixation, 2, 24-48.

7. Paerl, H. W., Tucker, C. S. (1995). Ecology of blue-green algae in aquaculture ponds. Journal of the World Aquaculture Society, 26(2), 109-131.

8. Sevrin-Reyssac, J., Pletikosic, M. (1990). Cyanobacteria in fish ponds. Aquaculture, 88(1), 1-20.

9. Reed, R. H., Stewart, W. D. P. (1985). Osmotic adjustment and organic solute accumulation in unicellular Cyanobacteria from freshwater and marine habitats. Marine Biology, 88(1), 1-9.

10. Murrell, M. C., Lores, E. M. (2004). Phytoplankton and zooplankton seasonal dynamics in a subtropical estuary: importance of Cyanobacteria. Journal of Plankton Research, 26(3), 371-382.

11. Sanchez-Baracaldo P. (2015). Origin of marine planktonic Cyanobacteria. Scientific reports, 5, 17418.

12. Stal, L. J. (2007). Cyanobacteria. In Algae and Cyanobacteria in extreme environments (pp. 659-680). Springer, Dordrecht.

13. Diez, B., Ininbergs, K. (2014). Ecological importance of Cyanobacteria. Cyanobacteria, 106, 41-63.

14. Desikachary, T.V. Taxonomy and Biology of Blue-green Algae. University of Madras, Madras, India, 1972.

15. Perumal, G.M., Anand, M. Manual of Freshwater algae of Tamil Nadu. Bishen Singh Mahendra Pal Singh, Dehra Dun, India, 2009. 